\title{
Massively Parallel Algorithms for Finding Well-Connected Components in Sparse Graphs
}

\author{
Sepehr Assadi ${ }^{\dagger}$ \\ sassadi@princeton.edu \\ Princeton University \\ Princeton, NJ, USA
}

\author{
Xiaorui Sun \\ xiaorui@uic.edu \\ University of Illinois \\ Chicago, IL, USA
}

\author{
Omri Weinstein \\ omri@cs.columbia.edu \\ Columbia University \\ New York, NY, USA
}

\begin{abstract}
Massively parallel computation (MPC) algorithms for graph problems have witnessed a resurgence of interest in recent years. Despite major progress for numerous graph problems however, the complexity of the sparse graph connectivity problem in this model has remained elusive: While classical logarithmic-round PRAM algorithms for finding connected components in any $n$-vertex graph have been known for more than three decades (and imply the same bounds for MPC model), no $o(\log n)$-round MPC algorithms are known for this task with truly sublinear in $n$ memory per machine (which is the only interesting regime for sparse graphs with $O(n)$ edges). It is conjectured that an $o(\log n)$-round algorithm for connectivity on general sparse graphs with $n^{1-\Omega(1)}$ per-machine memory may not exist, a conjecture that also forms the basis for multiple conditional hardness results on the round complexity of other problems in the MPC model.
\end{abstract}

We take an opportunistic approach towards the sparse graph connectivity problem by designing an algorithm with improved performance in terms of the connectivity structure of the input graph. Formally, we design an MPC algorithm that finds all connected components with spectral gap at least $\lambda$ in a graph in $O(\log \log n+\log (1 / \lambda))$ MPC rounds and $n^{\delta}$ memory per machine for any constant $\delta \in$ $(0,1)$. While this algorithm still requires $\Theta(\log n)$ rounds in the worst-case, it achieves an exponential round reduction on "wellconnected" components with $\lambda \geq 1 / \operatorname{polylog}(n)$ using only $n^{\delta}$ memory per machine and $\widetilde{O}(n)$ total memory, and still operates in $o(\log n)$ rounds even when $\lambda=1 / n^{o(1)}$

En-route to our main result, we design a new distributed data structure for performing independent random walks from all vertices simultaneously, as well as a new leader-election algorithm with exponentially faster round complexity on random graphs.

\footnotetext{
*A full version of the paper is available on arXiv.

${ }^{\dagger}$ Supported in part by the Simons Collaboration on Algorithms and Geometry. Majority of work done while the author was a graduate student at University of Pennsylvania.

Permission to make digital or hard copies of all or part of this work for personal or classroom use is granted without fee provided that copies are not made or distributed for profit or commercial advantage and that copies bear this notice and the full citation on the first page. Copyrights for components of this work owned by others than ACM must be honored. Abstracting with credit is permitted. To copy otherwise, or republish, to post on servers or to redistribute to lists, requires prior specific permission and/or a fee. Request permissions from permissions@acm.org.

PODC '19, July 29-August 2, 2019, Toronto, ON, Canada

(c) 2019 Association for Computing Machinery.

ACM ISBN 978-1-4503-6217-7/19/07 . \$ \$15.00

https://doi.org/10.1145/3293611.3331596
}

\section{CCS Concepts}

- Theory of computation $\rightarrow$ Massively parallel algorithms; Design and analysis of algorithms; Graph algorithms analysis.

\section{Keywords}

Sparse connectivity, spectral gap, random walk

\section{ACM Reference Format:}

Sepehr Assadi, Xiaorui Sun, and Omri Weinstein. 2019. Massively Parallel Algorithms for Finding Well-Connected Components in Sparse Graphs. In 2019 ACM Symposium on Principles of Distributed Computing (PODC '19), fuly 29-August 2, 2019, Toronto, ON, Canada. ACM, New York, NY, USA, 10 pages. https://doi.org/10.1145/3293611.3331596

\section{Introduction}

Recent years have witnessed a resurgence of interest in the theory of parallel computation, motivated by the successful deployment of parallel computing platforms such as MapReduce, Hadoop and Spark $[17,46,48]$. The massively parallel computation (MPC) model $[3,9,23,28]$ is a theoretical abstraction which aims to capture the design principles and main distinguishing aspects of these platform over the classical PRAM model: more local computation power (in principle unbounded), and larger local memory per processor. Consequently, it is typically possible to simulate a PRAM algorithm in the MPC model with no asymptotic blowup in the number of rounds $[23,28]$. However, these powerful features anticipate a potential for qualitatively smarter and dramatically faster parallel algorithms. A fundamental question on this front is then:

\section{How can the additional power of the MPC model be leveraged to achieve faster algorithms compared to clas- sical parallel models such as PRAM algorithms?}

The answer to this question turns out to be highly dependent on the type of problems at hand and the setting of parameters. For graph problems-the focus of this paper-the first improvement over PRAM algorithms was already achieved by Karloff et al. [28] who developed algorithms for graph connectivity and MST in $O(1)$ MPC rounds on machines with local memory $n^{1+\Omega(1)}$; here, $n$ is the number of vertices in the graph. This is in contrast to the $\Omega(\log n)$ round needed in the standard PRAM model for these problems (see, e.g., $[15,18,24,27,38,40,45])$. Since then, numerous algorithms have been designed for various graph problems that achieve $O(1)$ round-complexity with local memory $n^{1+\Omega(1)}$ on each machine (see, e.g., $[1,8,31,33]$ and references therein). 
The next set of improvements reduced the memory per machine to $\widetilde{O}(n)$. For example, an $O(1)$ round algorithm for MST and connectivity using only $O(n)$ memory per machine has been proposed in [26] building on previous work in [20, 25, 35] (see also [2, 10, 34] for related results). Another work is the recent $O(1)$ round algorithm of Assadi et al. [7] for $(\Delta+1)$-vertex coloring with $\widetilde{O}(n)$ memory per machine. A series of very recent papers $[5,6,12,16,19,30]$ have also achieved $O(\log \log n)$-round algorithms for different graph problems such as matching, vertex cover, and MIS in the MPC model, when the memory per machine is $O(n)$ or even $O(n / \operatorname{polylog}(n))$.

Most recently, several algorithms were also designed for matching, vertex cover, MIS, and $(\Delta+1)$ vertex coloring for the strongly sublinear memory regime, e.g. $n^{\delta}$ for any constant $\delta \in(0,1)$ memory per machine. In particular, for maximal matching and MIS on machines with $n^{\delta}$ memory, Behnezhad et al. [11] and Brandt et al. [13] gave $O(\log \log n)$-round MPC algorithms on bounded arboricity graphs, and Ghaffari and Uitto [21] gave an $O(\sqrt{\log n})$ round algorithm on general graphs (see also [37] for an algorithm for approximate matching and vertex cover with the same guarantee). An $O(\sqrt{\log \log n})$ round algorithm for $(\Delta+1)$ coloring in this regime was also given recently in [14].

Alas, this progress has came to a halt for the fundamental problem of connectivity on (sparse) undirected graphs. While classic $O(\log n)$-round PRAM algorithms for this problem have been known for more than three decades (see [18, 24, 27, 40, 45] and references therein), no faster MPC algorithm with truly sublinear $n^{1-\Omega(1)}$ memory per machine is known to date (see, e.g. [28, 29, 39]).

There are several substantial reasons for the lack of progress on this fascinating problem. On one hand, $\Omega(\log n)$ rounds are known to be necessary for a restricted class of (routing-style) MPC algorithms [9], and in fact it has been conjectured that this logarithmic barrier may be unavoidable for any MPC algorithm [3, 9, 39, 42]. This belief led to a series of recent results that used sparse connectivity as a hardness assumption for proving conditional lower bounds in the MPC model for other problems (see [3, 47] and references therein). On the other hand, it was observed by Roughgarden et al. [42] that proving any $\omega(1)$ lower bound on round complexity of connectivity would imply $\mathrm{NC}^{1} \subsetneq \mathbf{P}$, a major breakthrough that is well beyond the reach of current techniques.

In this paper we take an opportunistic approach to the sparse connectivity problem, which exploits the connectivity structure of the underlying graph. In particular, we use spectral gap as a quantitative measure of connectedness of a graph and design an algorithm for connectivity with improved performance guarantee depending on the spectral gap of the connected components of the underlying graph. For example, when connected components of the graph have large spectral gap, say $\Omega(1)$ or even $\Omega(1 /$ polylog $(n))$ (as in expanders), our algorithm only requires $O(\log \log n)$ MPC rounds while using $n^{\delta}$ memory per machine for constant $\delta \in(0,1)$ and $\widetilde{O}(n)$ total memory. We elaborate more on our results in Section 1.1.

Massively Parallel Computation Model. We adopt the most stringent model of modern parallel computation among [3, 9, 23, 28], the so-called Massively Parallel Computation (MPC) model of [9]. Let $N$ be the input size. It is assumed that the local memory on each machine and the number of machine should be at most $N^{1-\delta}$ for some constant $\delta>0$. Additionally, we are typically interested in algorithms with total memory $\widetilde{O}(N)$, i.e., proportional to the input size (within logarithmic factors) [3, 9], although total memories as large as $O\left(N^{2-2 \delta}\right)$ have been also considered in more relaxed variants [28]. The motivation behind these constraints is that the number of machines, and local memory of each machine should be much smaller than the input size to the problem since these frameworks are used to process large datasets.

In this model, computation proceeds in rounds. During a round each machine runs a local algorithm on the data assigned to the machine. No communication between machines is allowed during a round. Between rounds, machines are allowed to communicate so long as each machine send or receive a communication no more than its memory. Any data output from a machine must be computed locally from the data residing on the machine and initially the input data is distributed across machines adversarially. The final output also resides in a distributed fashion across the machines. The goal is to minimize the total number of rounds.

\subsection{Our Results and Techniques}

The spectral gap of a graph is defined to be the second eigenvalue of the normalized Laplacian associated with this graph. We use spectral gap as a measure of "connectedness" of a graph and design an opportunistic algorithm for connectivity with improved performance depending on the spectral gap of the underlying graph.

Theorem 1 (MAIn Result). There exists an MPC algorithm that with high probability identifies all connected components of any given sparse undirected $n$-vertex graph $G(V, E)$ with $\widetilde{O}(n)$ edges and a lower bound of $\lambda \in(0,1)$ on the spectral gap of its connected components.

For constant $\delta>0$, the algorithm can be implemented with $O\left(\frac{1}{\lambda^{2}} \cdot n^{1-\delta} \cdot \operatorname{polylog}(n)\right)$ machines each with $O\left(n^{\delta} \cdot \operatorname{polylog}(n)\right)$ memory, and in $O(\log \log n+\log (1 / \lambda))$ MPC rounds.

We note that Theorem 1 can be extended to the case when the algorithm is oblivious to the value of $\lambda$ and still manages to achieve an improved performance depending on $\lambda$ (see full version). We should also remark that the dependence of $O(\log (1 / \lambda))$ in Theorem 1 is best possible short of obtaining an $o(\log n)$-round algorithm for sparse connectivity in general graphs as $\lambda=1 / \operatorname{poly}(n)$ in any undirected graph.

Our result is most interesting in the case when spectral gap of (each connected component) of the graph is lower bounded by a constant or even $1 / \operatorname{polylog}(n)$, i.e., for graphs with "well-connected" components. Examples of such graphs include random graphs and expanders (see also $[22,36]$ for real-life examples in social networks). In this case, we obtain an algorithm with $\widetilde{O}(n)$ total memory and $n^{\delta}$ per-machine memory for constant $\delta \in(0,1)$ which can identify all connected components in only $O(\log \log n)$ rounds. Nevertheless, the algorithm in Theorem 1 still manages to achieve a non-trivial improvement even when the spectral gap is as small as $1 / n^{o(1)}$. Even in this case, the algorithm requires $o(\log n)$ MPC rounds (and total memory which is larger than the input size by only an $n^{o(1)}$ factor). 
We also prove an unconditional $\Omega\left(\log _{s} n\right)$-round lower bound for the promise problem of connectivity on sparse expanders on machines with memory $s$. This implies that the "full power" of the MPC model is indeed required to achieve our speedup, as with $s=$ polylog $(n)$ memory, $\Omega\left(\log _{s} n\right)=\tilde{\Omega}(\log n)$ rounds are needed even on sparse expanders (this result us presented in Section 8).

Finally, we note that a simple application of the toolkit we develop in proving our main result in Theorem 1 also implies the following theorem.

THEOREM 2. There exists an MPC algorithm that given any arbitrary graph with high probability identifies all connected components in $O(\log \log n)$ rounds on machines of memory $O(n / \operatorname{polylog}(n))$, or $O(\sqrt{\log n})$ rounds with $O\left(n / 2^{O(\sqrt{\log n})}\right)$ memory.

Theorem 2 is reminiscent of the recent set of results in $[5,6$, $12,16,19,30]$ on achieving similar guarantees for other graph problems such as matching and vertex cover in the mildly sublinear in $n$ per-machine memory regime. This result emphasizes the truly sublinear in $n$ regime, i.e., $n^{1-\Omega(1)}$ per-machine memory, as the "real" barrier to obtaining efficient algorithms for sparse connectivity with improved performance compared to PRAM algorithms.

Techniques. The first main technical ingredient of this work is a distributed data structure for performing and processing short independent random walks (proportional to the mixing time of each component) from all vertices of the graph simultaneously, whose construction takes logarithmic number of rounds in length of the walk. While implementing random walks in distributed and parallel settings is a well-studied problem (see, e.g., [24, 27, 43, 44] and references therein), the guarantee of our algorithm in achieving independent random walks across all vertices in a small number of rounds and total memory, departs from previous work (independence is crucial in the context of our algorithm). Achieving this stronger guarantee requires different tools, in particular, a method for "regularizing" our graph using a parallel implementation of the replacement product operation (see, e.g. [41]) that we design .

The second main technical ingredient of this work is a novel leader-election based algorithm for finding a spanning tree of a random graph. The key feature of this algorithm that distinguishes it from previous MPC algorithms for sparse connectivity (see, e.g., $[28,29,39])$ is that on random graphs, it provably requires only $O(\log \log n)$ MPC rounds as opposed to $\Omega(\log n)$ (we point out that [29] also analyzed their algorithms on random graphs (see Lemma 9), but even on random graphs their algorithm requires $\Theta\left(\log ^{2} n\right)$ rounds). Our algorithm achieves this exponential speedup by contracting quadratically larger components to a single vertex in each step, while "preserving the randomness" in the resulting contracted graph to allow for recursion.

\subsection{Recent Developments}

Independently and concurrently to our work, Andoni et al. [4] and Lacki et al. [32] have also studied MPC algorithms for connectivity with the goal of achieving improved performance on graphs with "better connectivity" structure. Andoni et al. [4] considered graphs with low diameter and developed an algorithm with $n^{\delta}$ memory per machine and $O\left(\log D \cdot \log \log _{N / n}(n)\right)$ rounds, where $D$ is the largest diameter of any connected component and $N=\Omega(m)$ is the total memory. Lacki et al. [32] considered random graphs (where each edge independently appears w.p. $\Omega(\log n / n)$ ) and give an $O(\log \log n)$ round algorithm on such graphs with $n^{\delta}$ memory per machine.

While our results subsume that of [32], they are incomparable to the results by [4]: even though in any graph $D=O(\log n / \lambda)$, the number of rounds in [4] is $O(\log D \log \log n)$ for the main setting of interest when the total memory is within logarithmic factors of input size (the typical requirement of the MPC model ${ }^{1}$ [3, 9]). As such, our algorithm achieves quadratically smaller round complexity when the spectral gap is large, i.e., is $\Omega(1)$ or even $\Omega(1 /$ polylog $(n))$ (as in random graphs and graphs with moderate expansion), while [4] achieve better performance on graphs with small spectral gap but not too-large diameter. We remark that our mildly-sublinear space algorithm for general graphs in Theorem 2 is entirely orthogonal to both [4] and [32]. We give a detailed comparison of our techniques with those of $[4,32]$ in the full version.

Organization. We start with some essential definitions in Section 2. Sections 3, 4, and 5 contain the three main steps of our algorithm and Section 6 show how to combine these results and prove Theorem 1 . Our mildly sublinear space algorithm for general graphs and our lower bound result appear in, respectively, Section 7 and Section 8. Due to space limitations, most proofs are postponed to the full version of the paper.

\section{Preliminaries}

Notation. For a graph $G(V, E)$, we define $V(G)=V$ and $E(G)=E$, and let $n:=|V(G)|$ and $m:=|E(G)|$. For any vertex $v \in V$, we use $d_{v}$ to denote the degree of $v$ in $G$. Throughout the paper, we assume without loss of generality that $d_{v} \geq 1$ for all vertices $v \in G$ (i.e., $G$ does not have isolated vertices). We say that a subset $C \subseteq V(G)$ is a component of $G$ if the induced subgraph of $G$ on $C$ is connected. We say that a partition $C=\left\{C_{1}, \ldots, C_{k}\right\}$ of $V(G)$ is a componentpartition iff every $C_{i}$ is a component of $G$.

For simplicity of exposition, we follow [16] in using the following concise notation for representing ranges: for a value $x$ and parameter $\varepsilon \geq 0$, we use $\llbracket x \pm \varepsilon \rrbracket$ to denote the range $[x-\varepsilon, x+\varepsilon]$ and further extend this notation to include expressions, e.g. $\llbracket(x \pm \varepsilon)^{2} \rrbracket$.

Almost regular graphs. Let $\Delta \geq 1$ be an integer and $\varepsilon>0$ be any parameter. We say that a graph $G(V, E)$ is $\llbracket(1 \pm \varepsilon) \Delta \rrbracket$-almostregular iff degree of any vertex in $V(G)$ belongs to $\llbracket(1 \pm \varepsilon) \Delta \rrbracket$. We refer to $\varepsilon$ as the discrepancy factor of the an almost-regular graph.

Spectral gap and mixing time. For an undirected graph $G(V, E)$ on $n$ vertices, we use $\mathcal{L}$ to denote the normalized Laplacian matrix of $G$. The spectral gap of $G$ is the second smallest eigenvalue of $\mathcal{L}$, denoted by $\lambda_{2}(G)$. It is well-known that a lazy random walk on a graph $G$ takes $O\left(\log n / \lambda_{2}(G)\right)$ steps to converge to its stationary distribution on a graph $G$ (this number of steps is referred to as the

\footnotetext{
${ }^{1}$ Minimizing total memory is critical in this context as the straightforward algorithm that computes the transitive closure of the graph by matrix multiplication (see [28]) achieves $O(\log D)$ rounds, subsuming both our results and [4]. This algorithm however requires $\Omega\left(n^{2}\right)$ total memory and hence does not adhere to the restrictions of the MPC model.
} 
mixing time). This is the main property of spectral gap that we use throughout the paper.

Random graphs. For any integers $n, d \geq 1$, we use $\mathbb{G}(n, d)$ to denote the distribution on random undirected graphs $G$ on $n$ vertices chosen by picking for each vertex $v \in V(G),\lfloor d / 2\rfloor$ outgoing edges $(u, v)$ for $v$ chosen uniformly at random (with replacement) from $V(G)$ and then removing the direction of edges. Note that this notion of a random graph is related but no identical to the more familiar family of Erdos-Renyi random graphs.

\section{Step 1: Regularization}

In this section, we show how to "preprocess" our graph in order to prepare it for the main steps of our algorithm. Roughly speaking, this step takes the original graph $G$ and turn it into a regular-graph without increasing its mixing time by much. Formally,

LEMMA 3.1. There exists an MPC algorithm that given any graph $G(V, E)$ computes another graph $H$ with the following properties with high probability:

(1) $|V(H)|=2 m$ and $H$ is $\Delta$-regular for some absolute constant $\Delta=O(1)$.

(2) There is a one-to-one correspondence between the connected components of $G$ and $H$.

(3) Let $H_{i}$ be a connected component of $H$ corresponding to the connected component $G_{i}$ of graph $G$. For any $\gamma<1$, $T_{\gamma}\left(H_{i}\right)=O\left(\frac{\log (n / \gamma)}{\lambda_{2}\left(G_{i}\right)}\right)$.

The algorithm can be implemented on $O\left(m^{1-\delta}\right)$ machines each with $O\left(m^{\delta}\right)$ memory and in $O\left(\frac{1}{\delta}\right)$ MPC rounds.

To prove Lemma 3.1, we use an approach based on the standard replacement product. Due to space limitations, we postpone this proof entirely to the full version of the paper.

\section{Step 2: Randomization}

We present the second step of our algorithm in this section. Roughly speaking, this step transforms each connected component of the graph into a "random graph" on the same set of vertices. Formally,

Lemma 4.1. Suppose $G(V, E)$ is any n-vertex $\Delta$-regular graph such that $T_{\gamma^{*}}\left(G_{i}\right) \leq T$ for $\gamma^{*}:=n^{-10}$ and for all connected component $G_{i}$ of $G$. There exists an MPC algorithm that given $G$ and integer $T$ computes another graph $H$ with the following properties with high probability:

(1) $V(H)=V(G),|E(H)|=O(n)$ and each connected component $G_{i}$ of $G$ corresponds to a connected component $H_{i}$ of $H$ on $V\left(H_{i}\right)=V\left(G_{i}\right)$.

(2) The connected component $H_{i}$ of $H$ is a random graph on $n_{i}=\left|V\left(H_{i}\right)\right|$ vertices sampled from the distribution $\mathbb{D}\left(H_{i}\right)$ such that $\left|\mathbb{D}\left(H_{i}\right)-\mathbb{G}\left(n_{i}, 100 \log n\right)\right|_{\text {tvd }} \leq n^{-8}$.

The algorithm can be implemented with $O\left(T^{2} \cdot n^{1-\delta} \cdot \Delta \log ^{2} n\right)$ machines each with $O\left(n^{\delta}\right)$ memory and in $O\left(\frac{1}{\delta} \cdot(\log T+\right.$ $\log \log n))$ MPC rounds.

To prove Lemma 4.1, we design a general algorithm for performing independent random walks in the MPC model which can be of independent of interest. Let $G(V, E)$ be a $\Delta$-regular graph and $W=\Delta^{-1} \cdot A$ be its random walk matrix (note that this is scalar product with $\Delta^{-1}$ as $G$ is $\Delta$-regular). For any vertex $u \in V$, and integer $t \geq 1$, the vector $W^{t} \cdot \boldsymbol{e}_{u}$ denotes the distribution of a random walk of length $t$ starting from $u$ where $\boldsymbol{e}_{u}$ is an $n$-dimensional vector which is all zero except for the entry $u$ which is one. We use $\mathcal{D}_{\mathrm{RW}}(u, t)=W^{t} \cdot \boldsymbol{e}_{u}$ to denote this distribution.

Theorem 3. There exists an MPC algorithm that given any $\triangle$ regular graph $G(V, E)$ and integert $\geq 1$, outputs a vector $\left(v_{1}, \ldots, v_{n}\right)$ such that:

$$
\left|\mathbb{D}\left(v_{1}, \ldots, v_{n}\right)-\bigotimes_{i=1}^{n} \mathcal{D}_{\mathrm{RW}}\left(u_{i}, t\right)\right|_{t v d} \leq n^{-10},
$$

where $\mathbb{D}\left(v_{1}, \ldots, v_{n}\right)$ denotes the distributions of the output vertices. The algorithm can be implemented with $O\left(t^{2} \cdot n^{1-\delta} \cdot \Delta \log n\right)$ machines each with $O\left(n^{\delta}\right)$ memory and in $O\left(\frac{1}{\delta} \cdot(\log t+\log \log n)\right)$ rounds.

Lemma 4.1 can be proven using Theorem 3. We postpone this proof to the full version and sketch the proof of Theorem 3 instead.

\subsection{Proof Sketch of Theorem 3}

We start by introducing a key data structure in our algorithm.

\section{Layered Graph}

A key component of our algorithm in Theorem 3 is the notion of a layered graph which we define in this section.

Definition 1 (LAyered Graph). For a graph $G(V, E)$ and integer $t \geq 1$, the layered graph $\mathcal{G}(G, t)$ of $G$ is defined as the following directed graph:

(1) Vertex-set: The vertex-set $\mathcal{V}$ of $\mathcal{G}$ is the set of all triples $(u, i, j) \in V \times[2 t] \times[t+1]$.

(2) Edge-set: There is a directed edge $(u, i, j) \rightarrow(v, \ell, k)$ in $\mathcal{G}$ whenever $(u, v) \in E$ and $k=j+1$ for all choice of $i$ and $\ell$.

For any vertex $\alpha=(u, i, j) \in \mathcal{V}$, we define $v(\alpha)=u \in V$. We partition the set of vertices $\mathcal{V}$ into $t+1$ sets $\mathcal{V}_{1}, \ldots, \mathcal{V}_{t+1}$ where the $j$-th set consists of all vertices $(u, i, j)$ for $u \in V$ and $i \in[2 t]$. We refer to each set $\mathcal{V}_{j}$ as a layer of the graph $\mathcal{G}$. It is immediate to see that $\mathcal{G}$ consists of $t+1$ layers and all edges in $\mathcal{G}$ are going from one layer to the next. Additionally, any vertex $u \in V$, contains $2 t$ "copies" in every layer. As such, any edge in $E$ is mapped to $t$ directed bi-cliques on the $2 \cdot 2 t$ copies of its endpoints between every two consecutive layers of $\mathcal{G}$.

Paths and walks in $\mathcal{G}$ and $G$ : The key property of the layered graph $\mathcal{G}$ is that any path starting from $\mathcal{V}_{1}$ and ending in $\mathcal{V}_{t+1}$ in $\mathcal{G}$ corresponds to a walk of length $t$ (but not necessarily a path) in $G$. More formally, consider a path $\mathcal{P}_{\alpha}=\alpha_{1}, \alpha_{2}, \ldots, \alpha_{t+1}$ where $\alpha=\alpha_{1} \in \mathcal{V}_{1}$. We can associate to $\mathcal{P}_{\alpha}$ a walk of length $t$ in $G$ starting from the vertex $v=v(\alpha)$, denoted by $W\left(\mathcal{P}_{\alpha}\right)$, in a straightforward way by traversing the vertices $u_{i}=v\left(\alpha_{i}\right)$ for $\alpha_{i} \in \mathcal{P}_{\alpha}$.

Sampled layered graph. We work with a random subgraph of the layered graph defined as follows: For any vertex in $\mathcal{G}$ independently, we pick exactly one of its outgoing edges uniformly at random to form a subgraph $\mathcal{G}_{\mathrm{s}}$, referred to as the sampled layered graph. 
As the out-degree of any vertex in $\mathcal{G S}_{\mathrm{S}}$ is exactly one, starting from any vertex $\alpha \in \mathcal{V}_{1}$, there is a unique path $\mathcal{P}_{\alpha}$ of length $t$ in $\mathcal{G}_{\mathrm{S}}$ from $\alpha$ to some vertex $\beta \in \mathcal{V}_{t+1}$. It is easy see that a path $\mathcal{P}_{\alpha}$ in $\mathcal{G}_{\mathrm{S}}$ corresponds to a random walk of length $t$ in $\mathcal{G}$ starting from the vertex $v(\alpha)$ and ending in $v(\beta)$.

In the rest of this section, we show that a sampled layered graph contains $\Omega(n)$ vertex disjoint paths from the first layer to the last one with high probability. Intuitively, this allows us to "extract" $\Omega(n)$ independent random walks from a sampled layered graph. We then use this fact to design our algorithm for simulating independent random walks from all vertices in $G$.

Define $\mathcal{V}_{1}^{*} \subseteq \mathcal{V}_{1}$ as the set of all vertices $(v, 1,1) \in \mathcal{V}_{1}$ for $v \in V$. The following lemma, whose proof is postponed to the full version, is key to our analysis.

Lemma 4.2. For any vertex $\alpha \in \mathcal{V}_{1}^{*}, \mathcal{P}_{\alpha}$ in $\mathcal{G}_{\mathrm{S}}$ is vertex disjoint from $\mathcal{P}_{\beta}$ for all $\beta \neq \alpha \in \mathcal{V}_{1}^{*}$, with probability at least $1 / 2$.

\section{A Parallel Random Walk Algorithm}

We start by presenting an algorithm with a weaker guarantee than our final random walk algorithm: in this algorithm only $\Omega(n)$ vertices are able to achieve a truly independent random walk destination; moreover, these vertices are unknown to the algorithm. We then present a subroutine for detecting these $\Omega(n)$ vertices. Finally, we combine these two subroutines to obtain our final algorithm.

Recall that for any vertex $u \in V(G)$ and integer $t \geq 1, \mathcal{D}_{\mathrm{RW}}(u, t)$ is the distribution of a random walk of length $t$ from $u$. We present the following algorithm.

SimpleRandomWalk $(G, t)$. An algorithm for performing a random walk of length $t$ from every vertex in a given graph $G$.

Output: For any vertex $u_{i} \in V(G)$, a vertex $v_{i} \in V(G)$ such that $v_{i} \sim \mathcal{D}_{\mathrm{RW}}\left(u_{i}, t\right)$

(1) Randomly sample a sampled subgraph $\mathcal{G}_{\mathrm{S}}$ from the layered graph $\mathcal{G}(G, t)$.

(a) Set $\mathcal{V}=V(G) \times[2 t] \times[t+1]$, and distribute the vertices of $\mathcal{V}$ to all the machines such that each machine contains $O\left(n^{\delta}\right)$ vertices.

(b) For every vertex $\alpha=(v, i, j) \in \mathcal{V}$ such that $j \leq t$ in parallel independently and uniformly sample a number $n_{\alpha}$ from $[\Delta]$ and $i_{\alpha}$ from $[2 t]$.

(c) Set $\mathcal{G}_{\mathrm{S}}$ to be empty initially.

(d) For every vertex $\alpha=(v, i, j) \in \mathcal{V}$ such that $j \leq t$ in parallel set $v_{\alpha}$ to be $n_{\alpha}$-th neighbor of $v$ in $G$, and add an edge from $\alpha$ to $\left(v_{\alpha}, i_{\alpha}, j+1\right)$ to $\mathcal{G}_{\mathrm{S}}$.

(2) For any vertex $\alpha \in \mathcal{G}_{\mathrm{S}}$, define $N_{0}(\alpha)=\beta$ where $(\alpha, \beta) \in$ $\mathcal{G S}_{\mathrm{S}}$ is the (only) outgoing edge of $\alpha$ in $\mathcal{G S}_{\mathrm{S}}$ (define $\beta=\perp$ if $\alpha$ belongs to $\mathcal{V}_{t+1}$ and hence has no outgoing edge).

(3) For $i=1$ to $\log t$ phases: For every $\alpha \in G_{\mathrm{S}}$ in parallel let $N_{i}(\alpha)=N_{i-1}\left(N_{i-1}(\alpha)\right)$ (assuming $N_{i-1}(\perp)=\perp$ ).

(4) For any $\alpha \in \mathcal{V}_{1}^{*}$, return $v=v\left(N_{\log t}(\alpha)\right)$ as the target of the vertex $u=v(\alpha)$ (recall that $\mathcal{V}_{1}^{*}$ is the set of all vertices $(u, 1,1) \in \mathcal{V}$ for $u \in V(G))$.
We first have the following simple claim.

CLAIm 4.3. For any vertex $\alpha \in \mathcal{V}_{1}^{*}$ of $\mathcal{G}_{\mathrm{S}}, N_{\log t}(\alpha)$ is the endpoint of the path $\mathcal{P}_{\alpha}$ in $\mathcal{G S}_{\mathrm{S}}$.

Proof. We prove by induction that $N_{i}(\alpha)$ is the vertex at distance $2^{i}$ from $\alpha$ in $\mathcal{P}_{\alpha}$. The base case for $i=0$ is true as $N_{0}(\alpha)=\beta$ where $\beta$ is the endpoint of the outgoing edge of $\alpha$. For $i>0$, by induction, $N_{i-1}(\alpha)$ is the vertex $\theta$ at distance $2^{i-1}$ from $\alpha$ and $N_{i-1}(\theta)$ is the vertex at distance $2^{i-1}$ from $\theta$. Hence $N_{i}(\alpha)=N_{i-1}\left(N_{i-1}(\alpha)\right)$ is at distance $2^{i}$ from $\alpha$ (as $\mathcal{G}_{\mathrm{S}}$ is a directed acyclic graph with edges going only from one layer to the next). As such, $N_{\log t}(\alpha)$ is at distance $t$ from $\alpha$ and hence is the endpoint of the path $\mathcal{P}_{\alpha}$.

We say that SimpleRandomWalk $(G, t)$ finds the vertex $v$ for $u$ if $v$ is returned as the target vertex of $u$. Claim 4.3 combined with the construction of sampled layered graph already implies that for any vertex $u \in V(G)$, the vertex $v$ found by SimpleRandomWalk is distributed according to $\mathcal{D}_{\mathrm{RW}}(u, t)$. We further have,

Lemma 4.4. For any vertex $u \in G$, SimpleRandomWalk $(G, t)$ finds a vertex $v \sim \mathcal{D}_{\mathrm{RW}}(u, t)$ such that with probability at least $1 / 2, v$ is independent of all other vertices found by SimpleRandomWalk.

Proof. Follows immediately from Claim 4.3 and Lemma 4.2 and the fact that random bits used in vertex disjoint paths of $\mathcal{G}$ s are independent of each other.

By Lemma 4.4, we are able to find $\Omega(n)$ independent random walks in $G$ with high probability. In the full version of the paper, we show that by running SimpleRandomWalk in parallel $O(\log n)$ times and combining them in $O(\log \log n)$ rounds, we can find a collection of independent random walks from every vertex of the graph with high probability.

\section{Step 3: Connectivity on Random Graphs}

In this section we present the final and paramount step of our algorithm, which involves finding connected components of a collection of disjoint random graphs chosen from $\mathbb{G}$.

LEMMA 5.1. Let $G(V, E)$ be a graph on $n$ vertices such that any connected component $G_{i}$ of $G$ with $n_{i}=\left|V\left(G_{i}\right)\right|$ is sampled from $\mathbb{G}\left(n_{i}, 100 \log n\right)$. There exists an MPC algorithm which identifies all connected components of $G$ with high probability (over the randomness of the algorithm and the distribution $\mathbb{G}$ ). For any $\delta>0$, the algorithm can be implemented with $O\left(n^{1-\delta}\right)$. $\operatorname{polylog}(n)$ machines each with $O\left(n^{\delta}\right) \cdot \operatorname{polylog}(n)$ memory and $O\left(\frac{1}{\delta} \cdot \log \log n\right) M P C$ rounds.

During the course of our exposition in this section, we need to set many parameters which we collect here for convenience.

- $\varepsilon:=(100 \cdot \log n)^{-2}$ : discrepancy of almost-regular graphs,

- $s:=\frac{10^{6} \cdot \log n}{\varepsilon^{2}}$ : scaler for degree of almost-regular graphs,

- $\Delta:=100 \cdot s:$ the degree of almost-regular graphs,

- $F:=\arg \min _{i}\left\{\Delta^{2^{i}} \geq n^{1 / 100}\right\}:$ number of phases.

Throughout this section, we define the degree of almost-regular graphs by multiplicative factors of $s$; this is needed to simplify many 
concentration bounds used in the proofs. We further point out that $F=O(\log \log n)$ and $\Delta^{F} \in\left[n^{1 / 100}, n^{1 / 50}\right]$ and hence $\Delta^{-F}=o(\varepsilon)$.

Preprocessing step. The first step in proving Lemma 5.1, is to make each connected component $G_{i}$ of $G$ "more random", i.e., turn it to a graph sampled from $\mathbb{G}$ with larger per-vertex degree. This can be easily done using Lemma 4.1 in previous section, as the graph $G_{i} \sim \mathbb{G}\left(n_{i}, 100 \log n\right)$ has a small mixing time (see full version).

Now consider the following preprocessing process: For $(F \cdot \Delta$. $s /(100 \log n))$ steps in parallel, we run the algorithm in Lemma 4.1 on the original graph $G$. For each connected component $G_{i}$ of $G$, this results us in having $F$ graphs $\widetilde{G}_{i, 1}, \ldots, \widetilde{G}_{i, F}$ which are (almost) sampled from the distribution $\mathbb{G}\left(n_{i}, \Delta \cdot s\right)$ (the distribution of these graphs is not exactly identical to this, but is rather close to this distribution in total variation distance which is sufficient for our purpose). As such, we now need to find the connected component of a graph $\widetilde{G}$ which is the union of all $\widetilde{G}_{i, j}$ for $i$ ranging over all connected components of $G$ and $j \in[F]$.

In the following lemma, we design an algorithm for this task. For simplicity of exposition, we state this lemma for the case of finding a spanning tree of one such connected component (i.e., assuming $G$ itself is sampled from $\mathbb{G}$ as opposed to having its connected components sampled from this distribution); however, it would be evident that running this algorithm on the original input results in finding a spanning tree of each connected component separately.

LEMMA 5.2. Let $\widetilde{G}$ be a graph on $n$ vertices such that $\widetilde{G}=\widetilde{G}_{1} \cup$ $\ldots \cup \widetilde{G}_{F}$ where $\widetilde{G}_{i} \sim \mathbb{G}(n, \Delta \cdot s)$. There exists an MPC algorithm that can find a spanning tree of $\widetilde{G}$ with high probability (over both the randomness of the algorithm and the distribution $\mathbb{G}$ ).

For any $\delta>0$, the algorithm can be implemented with $O\left(n^{1-\delta}\right)$. $\operatorname{polylog}(n)$ machines each with $O\left(n^{\delta}\right) \cdot \operatorname{polylog}(n)$ memory, and in $O\left(\frac{1}{\delta} \cdot \log \log n\right) M P C$ rounds.

The rest of this section is devoted to the proof of Lemma 5.2. At the end of the section, we use this lemma to prove Lemma 5.1. In this section, $n$ always refer to number of vertices in $\widetilde{G}$.

\subsection{Proof of Lemma 5.2}

We start with a simple definition.

Definition 2 (Contraction Graph). For a graph $G(V, E)$ and a partition $C:=\left\{C_{1}, \ldots, C_{k}\right\}$ of $V(G)$ (not necessarily a componentpartition), we construct a contraction graph $H$ of $G$ with respect to $C$ as the following graph:

(1) Vertex-set: The vertex-set $V(H)$ of $H$ is a collection ofk vertices where $w_{i} \in V(H)$ is labeled with the component $C_{i}$ of $C$, denoted by $C\left(w_{i}\right)$.

(2) Edge-set: For any $w \neq z \in V(H)$, there exists an edge $(w, z) \in$ $E(H)$ iff there exists vertices $u \in C(w)$ and $v \in C(z)$ where $(u, v) \in E(G)$ (H contains no parallel edges and no self-loops).

In other words, $H$ is obtained by "contracting" the vertices of $G$ inside each set of $C$ into a single vertex and removing parallel edges and self-loops.

Suppose $C$ is a component-partition of $G$ and $H$ is a contraction graph of $G$ with respect to $H$. Then it is immediate to see that we can construct a spanning tree (or forest) of $G$ given only spanning trees of each component in $C$ and a spanning tree of $H$.

Overview of the algorithm. The algorithm in Lemma 5.2 goes through $F$ phases. In each phase $i \in[F]$, it only considers the edges in $\widetilde{G}_{i}$ and use them to "grow" the components of $\widetilde{G}$ found in the previous phases. This part is done using a new leader-election algorithm that we design in this paper. This algorithm takes the contraction graph of $\widetilde{G}_{i}$ with respect to the set of components found already, and merge these components further to build larger components. The novelty of this leader-election algorithm is that starting from an (almost) $d$-regular graph, it can grow each component by a factor of (almost) $d$ (as opposed to typical leader-election algorithms that only increase size of each component by a constant factor).

Our main algorithm is then obtained by successively applying this leader election algorithm to contraction graph of $\widetilde{G}_{i}$ to build relatively large components of $\widetilde{G}_{i}$ and use them to refine the components found for $G$. The main step of our proof is to argue that if contraction graph of $\widetilde{G}_{i}$ was a random (almost) $d$-regular graph on $n^{\prime}$ vertices, then the contraction graph of $\widetilde{G}_{i+1}$ in this process would be another random (almost) $d^{2}$-regular graph on roughly $n^{\prime} / d$ vertices. Having achieved this, we can argue that each component of the graph $G$ grows by a quadratic factor in each phase, and hence after only $O(\log \log n)$ phase, each component has size $n^{\Omega(1)}$ (due to technical reasons, one cannot continue this argument until just one connected component of size $n$ remains). Finally, we prove that at this step, the diameter of the remaining graph, i.e., contraction of $G$ on the found components is only $O(1)$. A simple broadcasting algorithm can then be used to found a spanning tree of the remaining graph in $O(1)$ rounds.

\section{A Leader Election Algorithm}

We introduce a leader election algorithm, called LeaderElection $(H, d)$, which gets as an input an (almost) $(d \cdot s)$-regular graph and creates components of size (almost) $d$ in this graph.

LeaderElection $(H, d)$. A simple leader election algorithm for growing connected components on an (almost) $(d \cdot s)$-regular graph $H$.

(1) Set $L=\emptyset$ initially.

(2) For every vertex $v \in V(H)$ in parallel independently sample $p(v)$ from the Bernoulli distribution with probability $p:=s / d$ and insert $u$ to $L$ iff $p(v)=1$ (we refer to these vertices as leaders).

(3) Let $R:=V(H) \backslash L$.

(4) For any vertex $v \in R$ in parallel set $N_{L}(v)$ be the set of neighbors of $v$ in $L$ in graph $H$.

(5) For any vertex $v \in R$ in parallel let $M(v)$ be a vertex $u \in R$ chosen uniformly at random from $N_{L}(v)$ (we define $M(v)=\perp$ if $\left.N_{L}(v)=\emptyset\right)$.

(6) Return $k:=|L|$ sets $S_{v_{1}}, \ldots, S_{v_{k}}$ for $v_{1}, \ldots, v_{k} \in L$ such that $S_{v_{i}}=\left\{v_{i}\right\} \cup\left\{u \in R: M(u)=v_{i}\right\}$ (vertices with $M(u)=\perp$ are ignored). 
We have the following simple claim (see full version for proof).

CLAIm 5.3. Suppose in LeaderElection no vertex $v \in R$ has $M(v)=\perp$. Then, the collection $S_{1}, \ldots, S_{k}$ is a component-partition of $H$.

The main property of LeaderElection is that when computed on almost regular graphs it results in a component-partition with almost equal size components. In other words, if $H$ is a $\llbracket(1 \pm \varepsilon) d \cdot s \rrbracket$ almost-regular graph, then the resulting components are of size $\llbracket(1 \pm O(\varepsilon)) \cdot d \rrbracket$ each. The proof is deferred to the full version.

Lemma 5.4 (Equipartition Lemma). Let $\bar{\varepsilon} \in(\varepsilon, 1 / 100)$ and $H$ be $a \llbracket(1 \pm \bar{\varepsilon}) d \cdot s \rrbracket$-almost-regular graph for $d \geq s$. Then, with probability $1-1 / n^{23}$, for $\left(S_{1}, \ldots, S_{k}\right)=$ LeaderElection $(H, d)$ :

(1) For all $i \in[k],\left|S_{i}\right| \in \llbracket(1 \pm 3 \bar{\varepsilon}) d \rrbracket$,

(2) $\left(S_{1}, \ldots, S_{k}\right)$ is a component-partition of $V(H)$.

\section{Growing Connected Components}

We now use LeaderElection algorithm from the previous section to design our main algorithm which "grows" the size of connected components of $G$ repeatedly over $F$ phases.

GrowComponents $(\widetilde{G}, \Delta)$. An algorithm for "growing" connected components of size up to $n^{\Omega(1)}$ in a given graph $\widetilde{G}=\widetilde{G}_{1} \cup \ldots \cup \widetilde{G}_{F}$ where $\widetilde{G}_{i} \sim \mathbb{G}(n, \Delta \cdot s)$.

(1) Let $C_{1}$ be a partition of $V(\widetilde{G})$ into singleton sets.

(2) For $i=1$ to $F$ phases:

(a) Let $\Delta_{i}:=\Delta^{2^{i-1}}$ and $p_{i}=\Delta_{i}^{-1} \cdot s$.

(b) For every vertex $v \in V\left(\widetilde{G}_{i}\right)$ in parallel let $c_{i}(v)=j$ for $v \in C_{j}$.

(c) Construct contraction graph $H_{i}$ of $\widetilde{G}_{i}($ not $\widetilde{G})$ with respect to $C_{i}$ as follows:

(i) Set $H_{i}$ to be an empty set inititially.

(ii) For every edge $(u, v) \in E\left(\widetilde{G}_{i}\right)$ in parallel add $\left(C_{c_{i}(u)}, C_{c_{i}(v)}\right)$ to $H_{i}$.

(d) Compute $\left(S_{1}, \ldots, S_{k}\right)=$ LeaderElection $\left(H_{i}, \Delta_{i}\right)$ (hence, each $S_{j} \subseteq V\left(H_{i}\right)$ ).

(e) For each $S_{j}$ in parallel let $C_{i+1, j}=\bigcup_{w \in S_{j}} C_{i}(w)$.

(f) Let $C_{i+1}=\left\{C_{i+1,1}, \ldots, C_{i+1, k}\right\}$.

(3) Return the graph $H_{F}$.

We prove that for each phase $i \in[F]$, the contraction graph $H_{i}$ constructed in this phase is an almost-regular graph with degree roughly $\Delta_{i} \cdot s$ and discrepancy factor $\varepsilon_{i}:=\left(20^{i} \cdot \varepsilon\right)$. The following lemma is the heart of the proof.

LeMmA 5.5. In GrowComponents $(\widetilde{G}, \Delta)$, with high probability, for any $i \in[F]$ :

(I) $C_{i}$ is a component-partition of $\widetilde{G}$ with $\left|C_{i, j}\right| \in \llbracket\left(1 \pm \varepsilon_{i}\right) \Delta_{i} / \Delta \rrbracket$ for all $C_{i, j} \in C_{i}$.

(II) $H_{i}$ is $a \llbracket\left(1 \pm \varepsilon_{i}\right) \Delta_{i} \cdot s \rrbracket$-almost-regular graph on

$$
n_{i} \in \llbracket\left(1 \pm \varepsilon_{i}\right) \cdot n \Delta / \Delta_{i} \rrbracket
$$

vertices.
Proof. We prove this lemma inductively.

Base case: $C_{1}$ is clearly a component-partition of $\widetilde{G}$ as it only consists of singleton sets and $\left|C_{1, j}\right|=1$ for all $C_{1, j} \in C_{1}$. Since $\Delta_{1}=\Delta$, this proves the first part of the lemma in the base case. For the second part, as $C_{1}$ only consists of singleton sets, $H_{1}=\widetilde{G}_{1}$ and hence $n_{1}=n$. Finally, $H_{1}=\widetilde{G}_{1} \sim \mathbb{G}(n, \Delta \cdot s)$ and hence a simple Chernoff bound ensures that $H_{1}$ is a $\llbracket(1 \pm \varepsilon) \Delta \cdot s \rrbracket$-almost-regular graph, hence concluding the proof of the base case.

Induction step: Now suppose the lemma for $i>1$ and we prove it for $i+1$. By induction, we have that $H_{i}$ is a $\llbracket\left(1 \pm \varepsilon_{i}\right) \Delta_{i} \cdot s \rrbracket$-almostregular graph on $n_{i} \in \llbracket\left(1 \pm \varepsilon_{i}\right) \cdot n \cdot \Delta / \Delta_{i} \rrbracket$ vertices. In this phase, we compute $\left(S_{1}, \ldots, S_{k}\right)=$ LeaderElection $\left(H_{i}, \Delta_{i}\right)$. We can thus apply Lemma 5.4 with parameters $d=\Delta_{i}, p=p_{i}$, and $\bar{\varepsilon}=\varepsilon_{i}<1 / 100$, and obtain that with high probability,

$$
\left|S_{i}\right| \in \llbracket(1 \pm 3 \bar{\varepsilon}) \cdot \Delta_{i} \rrbracket=\llbracket\left(1 \pm 3 \varepsilon_{i}\right) \cdot \Delta_{i} \rrbracket,
$$

and $\left(S_{1}, \ldots, S_{k}\right)$ is a component-partition of $H_{i}$. In the following, we condition on this event.

Proof of part (I): Since $C_{i}$ is a component-partition of $\widetilde{G}$ (by induction), we have that vertices in $H_{i}$ correspond to components of $\widetilde{G}$, i.e., vertices in $C_{i}(w)$ for all $w \in V\left(H_{i}\right)$ are connected in $\widetilde{G}$. Moreover, by Lemma $5.4,\left(S_{1}, \ldots, S_{k}\right)$ is a component-partition of $H_{i}$ and hence vertices (of $H_{i}$ ) in each $S_{j}$ for $j \in[k]$ are connected to each other (in $H_{i}$ ). As edges of $H_{i}$ correspond to edges in $\widetilde{G}_{i} \subseteq \widetilde{G}$, any $C_{i+1, j} \in C_{i+1}$ is a component of $\widetilde{G}$, hence $C_{i+1}$ is a componentpartition of $\widetilde{G}$.

We now prove the bound on size of each $C_{i+1, j} \in C_{i+1}$. By definition,

$$
\begin{aligned}
\left|C_{i+1, j}\right| & =\sum_{w \in S_{j}}\left|C_{i}(w)\right| \in \llbracket\left|S_{j}\right| \cdot\left(1 \pm \varepsilon_{i}\right) \Delta_{i} / \Delta \rrbracket \\
& \subseteq \llbracket\left(\left(1 \pm 3 \varepsilon_{i}\right) \cdot \Delta_{i}\right) \cdot\left(\left(1 \pm \varepsilon_{i}\right) \Delta_{i} / \Delta\right) \rrbracket \quad(\text { by Eq }(1)) \\
& \subseteq \llbracket\left(1 \pm 5 \varepsilon_{i}\right) \cdot \Delta_{i}^{2} / \Delta \rrbracket=\llbracket\left(1 \pm 5 \varepsilon_{i}\right) \cdot \Delta_{i+1} / \Delta \rrbracket, \quad(2)
\end{aligned}
$$

as $\Delta_{i}^{2}=\Delta_{i+1}$. By the choice of $\varepsilon_{i+1}>5 \varepsilon_{i}$, this finalizes the proof of the first part. We now consider the second part.

Proof of part (II): Notice that $n_{i+1}=\left|C_{i+1}\right|$ as each set in $C_{i+1}$ is contracted to a single vertex in $H_{i+1}$. Since $C_{i+1}$ partitions $V(G)$, and as by Eq (2) each set in $C_{i+1}$ has size in $\llbracket\left(1 \pm 5 \varepsilon_{i}\right) \cdot \Delta_{i+1} / \Delta \rrbracket$, we have

$$
n_{i+1} \in \llbracket \frac{n}{\left(1 \pm 5 \varepsilon_{i}\right) \cdot \Delta_{i+1} / \Delta} \rrbracket \subseteq \llbracket\left(1 \pm 6 \varepsilon_{i}\right) n \cdot \Delta / \Delta_{i+1} \rrbracket .
$$

As $\varepsilon_{i+1}>6 \varepsilon_{i}$, this proves the bound on $n_{i+1}$. It remains to prove $H_{i+1}$ is an $\llbracket\left(1 \pm \varepsilon_{i+1}\right) \Delta_{i+1} \cdot s \rrbracket$-almost-regular graph. This is the main part of the argument.

LeMmA 5.6. For any vertex $w \in V\left(H_{i+1}\right)$, degree of $w$ in $H_{i+1}$ is $d_{w} \in \llbracket\left(1 \pm \varepsilon_{i+1}\right) \Delta_{i+1} \cdot s \rrbracket$ with high probability.

Proof. Recall that $H_{i+1}$ is a contraction graph of $\widetilde{G}_{i+1}$ with respect to the partition $C_{i+1}$. We define $C=C_{i+1}(w) \in C_{i+1}$. In $H_{i+1}, w$ has an edge to another vertex $z \in V\left(H_{i+1}\right)$ iff there exists a vertex $u \in C \subseteq V\left(\widetilde{G}_{i+1}\right)$ such that $u$ has an edge to some vertex $v \in C_{i+1}(z)$ in the graph $\widetilde{G}_{i+1}$. As such, degree of $w$ is equal to 
the number of sets $C_{i+1, j} \subseteq V\left(\widetilde{G}_{i+1}\right)$ such that there is an edge $(u, v) \in E\left(\widetilde{G}_{i+1}\right)$ for $u \in C$ and $v \in C_{i+1, j}$.

Now consider the process of generating $\widetilde{G}_{i+1} \sim \mathbb{G}(n, \Delta \cdot s)$ and notice that the edges chosen in $\widetilde{G}_{i+1}$ are chosen independent of the choice of $C_{i+1}$ as $C_{i+1}$ is only a function of the graphs $\widetilde{G}_{1}, \ldots, \widetilde{G}_{i}$. Moreover, recall that in $\mathbb{G}(n, \Delta \cdot s)$ each vertex chooses $\Delta \cdot s / 2$ other vertices uniformly at random to connect to (and then we remove the direction of edges). For any two sets $S, T \subseteq V\left(\widetilde{G}_{i+1}\right)$, we say that $S$ "hits" $T$ if there exists a vertex in $S$ which picks a directed edge to some vertex in $T$ in the process of generating $\widetilde{G}_{i+1}$ (so it is possible that $S$ hits $T$ but $T$ does not hit $S$ ). Let $K \subseteq[k]$ be such that for each $j \in K$, either $C$ hits $C_{i+1, j}$ or vice versa. By the above argument $d_{w} \in \llbracket|K| \pm 1 \rrbracket$ (to account for the fact that $C$ hitting $C$ does not change the degree of $w$ as we have no self-loops in $H_{i+1}$ ). In the following two claims, we bound $|K|$. We postpone the proofs of these two claims to the full version.

CLAIM 5.7. Let $K^{+} \subseteq[k]$ be the set of all indices $j \in[k]$ such that $C$ hits $C_{i+1, j}$. Then, with high probability, $\left|K^{+}\right| \in \llbracket\left(1 \pm \varepsilon_{i+1}\right) \Delta_{i+1} \cdot s / 2 \rrbracket$.

One interpretation of Claim 5.7 is that that distribution of $H_{i+1}$ is similar to $\mathbb{G}\left(n_{i+1}, \Delta_{i+1} \cdot s\right)$ with the difference that the number of out-edges chosen in $\mathbb{G}$ is not exactly $\Delta_{i+1} \cdot s$ (but quite close to it for each vertex). As such, we would expect $H_{i+1}$ to still behave similarly as $\mathbb{G}\left(n_{i+1}, \Delta_{i+1} \cdot s\right)$; in particular, be almost-regular with high probability.

CLAim 5.8. Let $K^{-} \subseteq[k]$ be the set of all indices $j \in[k] \backslash$ $K^{+}$such that $C_{i+1, j}$ hits $C$. Then, with high probability, $\left|K^{-}\right| \in$ $\llbracket\left(1 \pm \varepsilon_{i+1}\right) \Delta_{i+1} \cdot s / 2 \rrbracket$.

Lemma 5.6 now follows from Claims 5.7 and 5.8.

Lemma 5.6

To conclude the proof of Lemma 5.5, we simply take a union bound on all vertices $w \in V\left(H_{i+1}\right)$ and by Lemma 5.6 obtain that with high probability $d_{w} \in \llbracket\left(1 \pm \varepsilon_{i+1}\right) \Delta_{i+1} \cdot s \rrbracket$. This implies that $H_{i+1}$ is a $\llbracket\left(1 \pm \varepsilon_{i+1}\right) \Delta_{i+1} \cdot s \rrbracket$-almost regular graph, proving the induction step.

Finally, we claim that GrowComponents can also find a spanning tree of components in $C_{F}$.

Claim 5.9. Let $T$ be the set of edges chosen in LeaderElection (in defining $M(\cdot)$ for each non-leader vertex) in the course of execution of GrowComponents $(\widetilde{G}, \Delta)$. With high probability, the induced subgraph of $T$ on each component in $C_{F}$ is a spanning tree.

\section{Building the Spanning Tree}

Recall that by the choice of $F, \Delta_{F} \in\left[n^{1 / 100}, n^{1 / 50}\right]$. By running GrowComponents $(\widetilde{G}, \Delta)$, we obtain a graph $H_{F}$ which consists of $n_{F} \in \llbracket(1 \pm o(1)) \cdot n \cdot \Delta / \Delta_{F} \rrbracket$ vertices. Additionally, by Claim 5.7, with high probability, $H_{F}$ is a "random" graph (in spirit of $\mathbb{G}$ ) with $\llbracket(1 \pm o(1)) \Delta_{F} \rrbracket$ "out-degree" before removing the direction of edges. We use this to bound the diameter of $H_{F}$.

\section{Claim 5.10. Diameter of $H_{F}$ is $D=O(1)$ with high probability.}

We use the above claim to design a very simple algorithm to build a spanning tree of $H_{F}$. We then combine this algorithm with GrowComponents to prove Lemma 5.2.
Claim 5.11. Let $H$ be any graph with $m$ edges, $n$ vertices, and diameter D. A spanning tree of $H$ can be found in $O(D / \delta) M P C$ rounds with $O\left(m^{1-\delta}\right)$ machines with memory $O\left(m^{\delta}\right)$ for any $\delta>0$.

We are now ready to conclude the proof of Lemma 5.2.

Proof of Lemma 5.2. By Claim 5.9, we can find a spanning tree of every component of $\widetilde{G}$ in $C_{F}$. This step requires $O(\log \log n / \delta)$ MPC rounds on $\widetilde{O}\left(n^{1-\delta}\right)$ machines of memory $\widetilde{O}\left(n^{\delta}\right)($ as $|E(\widetilde{G})|=$ $\widetilde{O}(n)$ by construction). Note that the components in $C_{F}$ correspond to vertices of $H_{F}$ and hence by finding a spanning tree of $H_{F}$ we obtain a spanning tree of $\widetilde{G}$.

By Claim 5.10, diameter of $H_{F}$ is only $O(1)$. Hence, by the algorithm in Claim 5.11, we can find a spanning tree of $H_{F}$ in only $O(1 / \delta)$ rounds on machines of memory $O\left(n^{\delta}\right)$. Combining these trees, we obtain a spanning tree of $\widetilde{G}$, finalizing the proof.

\section{Putting Everything Together}

We now put all components of our algorithms in the previous three sections together and prove the following theorem which formalize Theorem 1 in the introduction.

THEOREM 4. There exists a randomized MPC algorithm that with high probability identifies all connected components of any given undirected $n$-vertex graph $G(V, E)$ with $m$ edges and a lower bound of $\lambda \in(0,1)$ on the spectral gap of any of its connected components. For any $\delta>0$, the algorithm can be implemented with $O\left(\frac{1}{\lambda^{2}} \cdot m^{1-\delta}\right.$. polylog $(n))$ machines each with $O\left(m^{\delta} \cdot \operatorname{polylog}(n)\right)$ memory, and in $O\left(\frac{1}{\delta} \cdot(\log \log n+\log (1 / \lambda))\right)$ MPC rounds.

Proof. We prove this theorem by applying the transformation steps in the previous sections to graph $G$.

Step 1. Let $G_{1}:=G$ with $n_{1}:=\left|V\left(G_{1}\right)\right|$ and $m_{1}:=\left|E\left(G_{1}\right)\right|$. We apply Lemma 3.1 to $G_{1}$ to obtain a $\Delta$-regular graph $G_{2}$ with the following properties (with high probability): there is a one-to-one correspondence between connected components of $G_{1}$ and $G_{2}$, and each connected component of $G_{2}$ has mixing time $T_{\gamma^{*}}=O(\log n / \lambda)$ with $\gamma^{*}=n^{-10}$. Moreover, $n_{2}:=\left|V\left(G_{2}\right)\right|=O\left(m_{1}\right)$ and $\Delta=O(1)$. By identifying connected components of $G_{2}$, we immediately identify connected components of $G_{1}$.

This step can be implemented in $O\left(m^{1-\delta}\right)$ machines with $O\left(m^{\delta}\right)$ memory in $O(1 / \delta)$ MPC rounds by Lemma 3.1 (as $m_{1}=m$ ).

Step 2. We apply Lemma 4.1 to $G_{2}$ with $T=T_{\gamma^{*}}$ to (with high probability) obtain a graph $G_{3}$ such that $V\left(G_{2}\right)=V\left(G_{3}\right)$ and for any connected component $G_{2, i}$ on $n_{2, i}$ vertices, the induced subgraph of $G_{3}$ on vertices $V\left(G_{2, i}\right)$, denoted by $G_{3, i}$, is a connected component of $G_{3}$ with distribution $\mathbb{D}\left(G_{3, i}\right)$ where

$$
\left|\mathbb{D}\left(G_{3, i}\right)-\mathbb{G}\left(n_{2, i}, 100 \log n\right)\right|_{t v d} \leq n^{-10} .
$$

Identifying connected components of $G_{2}$ is equivalent to identifying connected components of $G_{3}$.

This step can be implemented with $\widetilde{O}\left(n_{2}^{1-\delta}\right)$ machines with $\widetilde{O}\left(n_{2}^{\delta}\right)$ memory in $O((\log T+\log \log n) / \delta)$ rounds by Lemma 4.1 . Plugging in the value of these parameters, we obtain that this step 
is implementable with $\widetilde{O}\left(m^{1-\delta}\right)$ machines with $\widetilde{O}\left(m^{\delta}\right)$ memory in $O\left(\frac{1}{\delta}(\log \log n+\log (1 / \lambda))\right)$ MPC rounds.

Step 3. Let $n_{3}=n_{2}$ be the number of vertices in $G_{3}$. We apply Lemma 5.1 to $G_{3}$ to identify the connected components of $G$. The distribution of each connected component $G_{3, i}$ of $G_{3}$ is $\left(n^{-8}\right)$-close in total variation distance to $\mathbb{G}\left(n_{2, i}, 100 \log n\right)\left(n_{2, i}=\left|V\left(G_{3, i}\right)\right|\right)$. Hence, by the guarantee of Lemma 5.1, with high probability we are able to identify connected components of the graph $G_{3}$. This allows us to identify connected components of $G_{2}$ and $G_{1}=G$.

This step can be implemented in $\widetilde{O}\left(n_{3}^{1-\delta}\right)$ machines with $\widetilde{O}\left(n_{3}^{\delta}\right)$ memory in $O\left(\log \log n_{3} / \delta\right)$ rounds by Lemma 5.1. Plugging in the value of these parameters, we obtain that this step is implementable with $\widetilde{O}\left(m^{1-\delta}\right)$ machines with $\widetilde{O}\left(m^{\delta}\right)$ memory in $O(\log \log n / \delta)$ MPC rounds.

This concludes the proof of the theorem.

\section{A Mildly Sublinear Space Algorithm for Connectivity}

In this section, we present a simple algorithm for solving the sparse connectivity problem (in general, e.g., with no assumption on spectral gap, etc.) using $o(n)$ memory per-machine, proving Theorem 2 from introduction.

The algorithm in Theorem 2 is a simple application of the toolkit we developed for proving our main result in Theorem 4, combined with the linear-sketching algorithm of Ahn et al. [2] for graph connectivity. In particular, we use the following result from [2].

Proposition 7.1 ([2]). Let $H$ be any graph partitioned between $|V(H)|$ players such that each player receives all edges incident on a unique vertex in $V(H)$ (hence each edge is received by exactly two players). There exists a randomized algorithm in which every player sends a message of size $O\left(\log ^{3}|V(H)|\right)$ bits to a central coordinator who can output all connected components of $H$ using only these messages with high probability. The algorithm requires players to have access to polylog $(|V(H)|)$ shared random bits.

We are now ready to present the algorithm in Theorem 2.

SublinearConn $(G)$. A mildly sublinear space algorithm for connectivity on a given graph $G$.

(1) Set $d:=\frac{n \cdot(\log n)^{4}}{s}$ and $t:=\left(d^{3} \cdot 100 \log n\right)$, and run SimpleRandomWalk $(G, t)$.

(2) Create a graph $\widetilde{G}$ from $G$ by connecting every vertex $v \in$ $V(G)$ to all distinct vertices visited in the random walk starting from $v$ computed in the previous step.

(3) Run LeaderElection $(\widetilde{G}, d)$ and let $H$ be the graph obtained by contracting any component found by LeaderElection to a single vertex.

(4) Remove self-loops and duplicate edges from $H$ and run the algorithm in Proposition 7.1 on $H$ by using a dedicated machine to simulate a single player.

Theorem 2 can then be proven using SublinearConn algorithm as we show in the full version of the paper.

\section{An Unconditional Lower Bound}

We prove an unconditional lower bound on the number of MPC rounds required to solve the connectivity problem on sparse undirected graphs with a constant spectral gap. More formally, we henceforth denote by ExpanderConn ${ }_{n}$ the decision promise problem of determining connectivity on $n$-vertex graphs $G$, where in both cases (each connected component of) $G$ is guaranteed to be a sparse expander $(|E(G)|=O(n)$ and the spectral gap of each component is $\left.\lambda_{2}=\Omega(1)\right)$.

THEOREM 5 (LOWER BOUND FOR EXPANDER CONNECTIVITY). Every MPC algorithm for ExpanderConn ${ }_{n}$ with s space per machine (and an arbitrary number of machines) requires $r=\Omega\left(\log _{s} n\right)$ rounds of computation. This holds even against randomized MPC protocols with constant error probability.

Proof of Theorem 5 uses a characterization result of [42] for MPC algorithms and appears in the full version of the paper.

We remark that Theorem 5 suggests that any MPC algorithm with polylog $(n)$ memory per machine for connectivity even on union of expander graphs requires $\Omega(\log n / \log \log n)$ MPC rounds. In Remark 8.1, we further show a similar situation for (EREW) PRAM algorithms. Moreover, by a result of [42], the lower bound in Theorem 5 is asymptotically the best possible unconditional lower bound short of proving that $\mathrm{NC}^{1} \subsetneq \mathbf{P}$ which would be a major breakthrough in complexity theory.

REMARK 8.1 (Extension to THE PRAM MODEL). In Theorem 5 we proved the lower bound for ExpanderConn ${ }_{n}$ in the MPC model as our main focus in this paper is on this model after all. However, our proof of Theorem 5 implies that ExpanderConn ${ }_{n}$ requires $\Omega(\log n)$ rounds in the (EREW) PRAM model as well. The reason is that our proof implies ExpanderConn ${ }_{n}$ is a critical function of $\Omega(n / \log n)$ variables: its output depends on the existence or nonexistence of the $k=\Omega(n / \log n)$ expanders graphs $B_{1}, \ldots, B_{k}$ (think of ExpanderConn $n_{n}$ as OR function of $k$ bits, each denoting whether the $i$-th expander $B_{i}$ is present in $G$ or not). By results of $[15,38]$ this requires $\Omega(\log n)$ rounds in the (EREW) PRAM model.

\section{Acknowledgments}

We thank Alex Andoni, Michael Kapralov, Sanjeev Khanna, and Krzysztof Onak for helpful discussions. We are particularly grateful to Krzysztof Onak for pointing out a flaw in our original argument on the correctness of our random walk data structure that is fixed in the current version.

\section{References}

[1] Kook Jin Ahn and Sudipto Guha. 2015. Access to Data and Number of Iterations: Dual Primal Algorithms for Maximum Matching under Resource Constraints. In Proceedings of the 27th ACM on Symposium on Parallelism in Algorithms and Architectures, SPAA 2015, Portland, OR, USA, fune 13-15, 2015. 202-211.

[2] Kook Jin Ahn, Sudipto Guha, and Andrew McGregor. 2012. Analyzing Graph Structure via Linear Measurements. In Proceedings of the Twenty-third Annual ACM-SIAM Symposium on Discrete Algorithms (SODA '12). SIAM, 459-467. http: //dl.acm.org/citation.cfm?id=2095116.2095156

[3] Alexandr Andoni, Aleksandar Nikolov, Krzysztof Onak, and Grigory Yaroslavtsev. 2014. Parallel algorithms for geometric graph problems. In Symposium on Theory of Computing, STOC 2014, New York, NY, USA, May 31 - June 03, 2014. 574-583.

[4] Alexandr Andoni, Zhao Song, Clifford Stein, Zhengyu Wang, and Peilin Zhong. 2018. Parallel Graph Connectivity in Log Diameter Rounds. In 59th IEEE Annual 
Symposium on Foundations of Computer Science, FOCS 2018, Paris, France, October 7-9, 2018. 674-685.

[5] Sepehr Assadi. 2017. Simple Round Compression for Parallel Vertex Cover. CoRR abs/1709.04599 (2017)

[6] Sepehr Assadi, MohammadHossein Bateni, Aaron Bernstein, Vahab S. Mirrokni, and Cliff Stein. 2019. Coresets Meet EDCS: Algorithms for Matching and Vertex Cover on Massive Graphs. In Proceedings of the Thirtieth Annual ACM-SIAM Symposium on Discrete Algorithms, SODA 2019, San Diego, California, USA, January 6-9, 2019. 1616-1635.

[7] Sepehr Assadi, Yu Chen, and Sanjeev Khanna. 2019. Sublinear Algorithms for $(\Delta+$ 1) Vertex Coloring. In Proceedings of the Thirtieth Annual ACM-SIAM Symposium on Discrete Algorithms, SODA 2019, San Diego, California, USA, January 6-9, 2019. 767-786.

[8] Sepehr Assadi and Sanjeev Khanna. 2017. Randomized Composable Coresets for Matching and Vertex Cover. In Proceedings of the 29th ACM Symposium on Parallelism in Algorithms and Architectures, SPAA 2017, Washington DC, USA, fuly 24-26, 2017. 3-12.

[9] Paul Beame, Paraschos Koutris, and Dan Suciu. 2013. Communication steps for parallel query processing. In Proceedings of the 32nd ACM SIGMOD-SIGACT SIGART Symposium on Principles of Database Systems, PODS 2013, New York, NY, USA - fune 22 - 27, 2013. 273-284

[10] Soheil Behnezhad, Mahsa Derakhshan, and MohammadTaghi Hajiaghayi. 2018 Brief Announcement: Semi-MapReduce Meets Congested Clique. CoRR abs/1802.10297 (2018).

[11] Soheil Behnezhad, Mahsa Derakhshan, MohammadTaghi Hajiaghayi, and Richard M. Karp. 2018. Massively Parallel Symmetry Breaking on Sparse Graphs: MIS and Maximal Matching. CoRR abs/1807.06701 (2018).

[12] Soheil Behnezhad, MohammadTaghi Hajiaghayi, and David G. Harris. 2019 Exponentially Faster Massively Parallel Maximal Matching. CoRR abs/1901.03744 (2019).

[13] Sebastian Brandt, Manuela Fischer, and Jara Uitto. 2018. Matching and MIS for Uniformly Sparse Graphs in the Low-Memory MPC Model. CoRR abs/1807.05374 (2018).

[14] Yi-Jun Chang, Manuela Fischer, Mohsen Ghaffari, Jara Uitto, and Yufan Zheng 2018. The Complexity of (Delta+1) Coloring inCongested Clique, Massively Parallel Computation, and Centralized Local Computation. arXiv preprint arXiv:1808.08419 (2018)

[15] Stephen A. Cook, Cynthia Dwork, and Rüdiger Reischuk. 1986. Upper and Lower Time Bounds for Parallel Random Access Machines without Simultaneous Writes. SIAM 7. Comput. 15, 1 (1986), 87-97.

[16] Artur Czumaj, Jakub Lacki, Aleksander Madry, Slobodan Mitrovic, Krzysztof Onak, and Piotr Sankowski. 2018. Round compression for parallel matching algorithms. In Proceedings of the 50th Annual ACM SIGACT Symposium on Theory of Computing, STOC 2018, Los Angeles, CA, USA, June 25-29, 2018. 471-484.

[17] Jeffrey Dean and Sanjay Ghemawat. 2004. MapReduce: Simplified Data Processing on Large Clusters. In Proceedings of the 6th Conference on Symposium on Opearting Systems Design \& Implementation - Volume 6 (OSDI'04). USENIX Association, Berkeley, CA, USA, 10-10. http://dl.acm.org/citation.cfm?id=1251254.1251264

[18] Hillel Gazit. 1986. An optimal randomized parallel algorithm for finding connected components in a graph. In Foundations of Computer Science, 1986., 27th Annual Symposium on. IEEE, 492-501.

[19] Mohsen Ghaffari, Themis Gouleakis, Slobodan Mitrovic, and Ronitt Rubinfeld. 2018. Improved Massively Parallel Computation Algorithms for MIS, Matching, and Vertex Cover. CoRR abs/1802.08237 (2018)

[20] Mohsen Ghaffari and Merav Parter. 2016. MST in Log-Star Rounds of Congested Clique. In Proceedings of the 2016 ACM Symposium on Principles of Distributed Computing, PODC 2016, Chicago, IL, USA, July 25-28, 2016. 19-28.

[21] Mohsen Ghaffari and Jara Uitto. 2019. Sparsifying Distributed Algorithms with Ramifications in Massively Parallel Computation and Centralized Local Computation. In Proceedings of the Thirtieth Annual ACM-SIAM Symposium on Discrete Algorithms, SODA 2019, San Diego, California, USA, Fanuary 6-9, 2019. 1636-1653.

[22] Christos Gkantsidis, Milena Mihail, and Amin Saberi. 2003. Conductance and congestion in power law graphs. In Proceedings of the International Conference on Measurements and Modeling of Computer Systems, SIGMETRICS 2003, June 9-14, 2003, San Diego, CA, USA. 148-159.

[23] Michael T. Goodrich, Nodari Sitchinava, and Qin Zhang. 2011. Sorting, Searching, and Simulation in the MapReduce Framework. In Algorithms and Computation22nd International Symposium, ISAAC 2011, Yokohama, Japan, December 5-8, 2011. Proceedings. 374-383.

[24] Shay Halperin and Uri Zwick. 1994. An Optimal Randomized Logarithmic Time Connectivity algorithm for the EREW PRAM (Extended Abstract). In SPAA. 1-10.

[25] James W. Hegeman, Gopal Pandurangan, Sriram V. Pemmaraju, Vivek B. Sardeshmukh, and Michele Scquizzato. 2015. Toward Optimal Bounds in the Congested Clique: Graph Connectivity and MST. In Proceedings of the 2015 ACM Symposium on Principles of Distributed Computing, PODC 2015, Donostia-San Sebastián, Spain, Fuly $21-23,2015.91-100$.

[26] Tomasz Jurdzinski and Krzysztof Nowicki. 2018. MST in O(1) Rounds of Congested Clique. In Proceedings of the Twenty-Ninth Annual ACM-SIAM Symposium on Discrete Algorithms, SODA 2018, New Orleans, LA, USA, fanuary 7-10, 2018. 2620-2632.

[27] David R. Karger, Noam Nisan, and Michal Parnas. 1992. Fast Connected Components Algorithms for the EREW PRAM. In Proceedings of the Fourth Annual ACM Symposium on Parallel Algorithms and Architectures (SPAA '92). ACM, New York, NY, USA, 373-381. https://doi.org/10.1145/140901.141920

[28] Howard J. Karloff, Siddharth Suri, and Sergei Vassilvitskii. 2010. A Model of Computation for MapReduce. In Proceedings of the Twenty-First Annual ACMSIAM Symposium on Discrete Algorithms, SODA 2010, Austin, Texas, USA, Fanuary 17-19, 2010. 938-948.

[29] Raimondas Kiveris, Silvio Lattanzi, Vahab S. Mirrokni, Vibhor Rastogi, and Sergei Vassilvitskii. 2014. Connected Components in MapReduce and Beyond. In Proceedings of the ACM Symposium on Cloud Computing, Seattle, WA, USA, November 03 - 05, 2014. 18:1-18:13.

[30] Christian Konrad. 2018. MIS in the Congested Clique Model in $\mathrm{O}(\log \log \Delta)$ Rounds. CoRR abs/1802.07647 (2018).

[31] Ravi Kumar, Benjamin Moseley, Sergei Vassilvitskii, and Andrea Vattani. 2013. Fast greedy algorithms in mapreduce and streaming. In 25th ACM Symposium on Parallelism in Algorithms and Architectures, SPAA '13, Montreal, QC, Canada fuly 23 - 25, 2013. 1-10.

[32] Jakub Lacki, Vahab S. Mirrokni, and Michal Wlodarczyk. 2018. Connected Components at Scale via Local Contractions. CoRR abs/1807.10727 (2018).

[33] Silvio Lattanzi, Benjamin Moseley, Siddharth Suri, and Sergei Vassilvitskii. 2011. Filtering: a method for solving graph problems in MapReduce. In SPAA 2011: Proceedings of the 23rd Annual ACM Symposium on Parallelism in Algorithms and Architectures, San fose, CA, USA, June 4-6, 2011 (Co-located with FCRC 2011). 85-94. https://doi.org/10.1145/1989493.1989505

[34] Christoph Lenzen. 2013. Optimal deterministic routing and sorting on the congested clique. In ACM Symposium on Principles of Distributed Computing, PODC '13, Montreal, QC, Canada, fuly 22-24, 2013. 42-50.

[35] Zvi Lotker, Elan Pavlov, Boaz Patt-Shamir, and David Peleg. 2003. MST construction in $\mathrm{O}(\log \log \mathrm{n})$ communication rounds. In SPAA 2003: Proceedings of the Fifteenth Annual ACM Symposium on Parallelism in Algorithms and Architectures, June 7-9, 2003, San Diego, California, USA (part of FCRC 2003). 94-100.

[36] Fragkiskos D. Malliaros and Vasileios Megalooikonomou. 2011. Expansion Properties of Large Social Graphs. In Database Systems for Adanced Applications 16th International Conference, DASFAA 2011, International Workshops: GDB, SIM3, FlashDB, SNSMW, DaMEN, DQIS, Hong Kong, China, April 22-25, 2011. Proceedings. 311-322.

[37] Krzysztof Onak. 2018. Round Compression for Parallel Graph Algorithms in Strongly Sublinear Space. CoRR abs/1807.08745 (2018).

[38] Ian Parberry and Pei Yuan Yan. 1991. Improved Upper and Lower Time Bounds for Parallel Random Access Machines Without Simultaneous Writes. SIAM 7. Comput. 20, 1 (1991), 88-99.

[39] Vibhor Rastogi, Ashwin Machanavajjhala, Laukik Chitnis, and Anish Das Sarma. 2013. Finding connected components in map-reduce in logarithmic rounds. In 29th IEEE International Conference on Data Engineering, ICDE 2013, Brisbane, Australia, April 8-12, 2013. 50-61.

[40] JH Reif. 1985. Optimal parallel algorithms for interger sorting and graph connectivity. Technical report. Technical Report. Harvard Univ., Cambridge, MA (USA). Aiken Computation Lab.

[41] Omer Reingold, Salil P. Vadhan, and Avi Wigderson. 2000. Entropy Waves, the Zig-Zag Graph Product, and New Constant-Degree Expanders and Extractors. In 41st Annual Symposium on Foundations of Computer Science, FOCS 2000, 12-14 November 2000, Redondo Beach, California, USA. 3-13.

[42] Tim Roughgarden, Sergei Vassilvitskii, and Joshua R. Wang. 2016. Shuffles and Circuits: (On Lower Bounds for Modern Parallel Computation). In Proceedings of the 28th ACM Symposium on Parallelism in Algorithms and Architectures, SPAA 2016, Asilomar State Beach/Pacific Grove, CA, USA, July 11-13, 2016. 1-12.

[43] Atish Das Sarma, Sreenivas Gollapudi, and Rina Panigrahy. 2011. Estimating PageRank on graph streams. F. ACM 58, 3 (2011), 13:1-13:19.

[44] Atish Das Sarma, Danupon Nanongkai, Gopal Pandurangan, and Prasad Tetali. 2013. Distributed Random Walks. F. ACM 60, 1 (2013), 2:1-2:31.

[45] Yossi Shiloach and Uzi Vishkin. 1982. An O (logn) parallel connectivity algorithm. Journal of Algorithms 3, 1 (1982), 57-67.

[46] Tom White. 2009. Hadoop: The Definitive Guide (1st ed.). O'Reilly Media, Inc

[47] Grigory Yaroslavtsev and Adithya Vadapalli. 2017. Massively Parallel Algorithms and Hardness for Single-Linkage Clustering Under $\ell_{p}$-Distances. arXiv preprint arXiv:1710.01431 (2017)

[48] Matei Zaharia, Mosharaf Chowdhury, Michael J. Franklin, Scott Shenker, and Ion Stoica. 2010. Spark: Cluster Computing with Working Sets. In Proceedings of the 2Nd USENIX Conference on Hot Topics in Cloud Computing (HotCloud'10). USENIX Association, Berkeley, CA, USA, 10-10. http://dl.acm.org/citation.cfm? id $=1863103.1863113$ 